\title{
The joint association of physical activity and glycaemic control in predicting cardiovascular death and all-cause mortality in the US population
}

\author{
J. I. Reddigan • M. C. Riddell • J. L. Kuk
}

Received: 19 July 2011 / Accepted: 28 October 2011 /Published online: 13 November 2011

(C) Springer-Verlag 2011

\begin{abstract}
Aims/hypothesis The aim of this study was to examine the joint association of physical activity and glycaemic control as measured by $\mathrm{HbA}_{1 \mathrm{c}}$ on all-cause and cardiovascular disease (CVD) mortality risk.

Methods The sample included 10,352 adults from the Third National Health and Nutrition Examination Survey (NHANES III) Linked Mortality Public-use File (followup $13.4 \pm 3.9$ years; 2,463 deaths). Physical activity was assessed by questionnaire and classified into inactive and active categories based on self-reported frequency of leisure-time activity. $\mathrm{HbA}_{1 \mathrm{c}}$ was categorised to reflect the American Diabetes Association diagnostic and treatment guidelines.

Results Being physically active was associated with a decreased risk of all-cause (HR 0.74 [95\% CI 0.67, 0.81]) and CVD (HR 0.71 [95\% CI 0.62, 0.82]) mortality, whereas higher levels of $\mathrm{HbA}_{1 \mathrm{c}}$ were associated with an increased mortality risk. $\mathrm{HbA}_{1 \mathrm{c}} \geq 7 \%$ (53 $\left.\mathrm{mmol} / \mathrm{mol}\right)$ was associated with the highest risk for all-cause (HR 1.54 [95\% CI 1.30, 1.82]) and CVD (HR 1.93 [95\% CI 1.52, 2.45]) mortality.
\end{abstract}

Electronic supplementary material The online version of this article (doi:10.1007/s00125-011-2374-3) contains peer-reviewed but unedited supplementary material, which is available to authorised users.

J. I. Reddigan · J. L. Kuk $(\bowtie)$

School of Kinesiology and Health Science,

2002 Sherman Health Science Centre, York University,

4700 Keele Street,

Toronto, ON, Canada M3J 1P3

e-mail: jennkuk@yorku.ca

M. C. Riddell

School of Kinesiology and Health Science, York University,

Norman Bethune College,

Toronto, ON, Canada
Across all categories of $\mathrm{HbA}_{1 \mathrm{c}}$, active individuals were not at increased risk for all-cause mortality compared with inactive individuals with normal glycaemic control. Similar findings were observed for CVD mortality, except that active individuals with $\mathrm{HbA}_{1 \mathrm{c}} \geq 7 \%(53 \mathrm{mmol} / \mathrm{mol})$ were still at increased risk for CVD mortality. However, their risk for CVD death was substantially lower than the risk for their inactive counterparts (HR 1.38 [95\% CI 1.03, 1.84] vs HR 1.98 [95\% CI 1.34, 2.92]).

Conclusions/interpretation Physical activity is associated with lower all-cause and CVD mortality risk for individuals across all levels of glycaemic control. Therefore, engaging in a physically active lifestyle and achieving normal levels of glycaemic control may both be important for the prevention of early mortality.

Keywords Epidemiology $\cdot \mathrm{HbA}_{1 \mathrm{c}} \cdot$ Mortality risk $\cdot$ Physical activity
Abbreviations
$\mathrm{CDC}$
CVD
Centers for Disease Control and Prevention
NHANES III
Cardiovascular disease
Third National Health and Nutrition
Examination Survey

\section{Introduction}

Any degree of dysglycaemia, as measured by fasting glycaemia or $\mathrm{HbA}_{1 \mathrm{c}}$, is an established risk factor for various cardiovascular disease (CVD) outcomes including ischaemic and haemorrhagic stroke, myocardial infarction and death $[1,2]$. Although regular exercise is established as being beneficial for improving glycaemic control and 
various health outcomes in diabetes [3], the joint association of physical activity and glycaemic control on mortality risk is unclear. The purpose of this analysis is to examine the protective effect of physical activity on all-cause and CVD mortality risk in individuals across all levels of glycaemic control as measured by $\mathrm{HbA}_{1 \mathrm{c}}$ in the US population.

\section{Research design and methods}

Study design and population The study sample was obtained from the Third National Health and Nutrition Examination Survey (NHANES III), which is a nationally representative study of 33,994 US citizens between 1988 and 1994, with mortality follow-up through 31 December 2006. Cause of death was assessed using ICD9 and -10 codes. All participants gave their informed written consent before participation in the examination, and the study protocol was approved by the National Center for Health Statistics. Individuals were excluded if they were pregnant, had a BMI of less than $18.5 \mathrm{~kg} / \mathrm{m}^{2}$, were less than 20 years of age or had missing data for metabolic variables or physical activity. This left a final sample of 10,352 men and women.

Measurements At baseline, participants completed a questionnaire regarding the frequency of their participation over the preceding month in the following leisure-time activities: walking, jogging, bicycling, swimming, aerobics or aerobic dancing, other dancing, calisthenics or exercises, gardening or yard work, and weight lifting. Participants could also list up to four additional activities. Individuals reporting no leisure-time activity were classified as 'inactive' and individuals who reported engaging in a leisure-time activity one or more times per week were classified as being 'active'. Work and transportation physical activity was not assessed.

Age, sex, income, ethnicity, smoking status, alcohol intake, dietary fat intake $(>30 \%$ or $\leq 30 \%$ of kilocalories from fat), medication use (type 2 diabetes, CVD, dyslipidaemia or hypertension) and a self-reported physician diagnosis of hypertension, heart attack, stroke and congestive heart failure were also assessed by questionnaire. The physical examination included measurement of height, weight and blood pressure, and a fasting blood draw for assessment of glucose, insulin, total cholesterol, HDL cholesterol, $\mathrm{HbA}_{1 \mathrm{c}}$ and insulin resistance (HOMA). HOMA was derived from the following formula: (fasting insulin [pmol/1]/6) $\times($ fasting glucose $[\mathrm{mmol} / \mathrm{l}] / 22.5)[4] . \mathrm{HbA}_{1 \mathrm{c}}$ was classified as $<5.7 \%(39 \mathrm{mmol} / \mathrm{mol}), 5.7-6.4 \%$ (39$46 \mathrm{mmol} / \mathrm{mol}), 6.5-6.9 \%(48-52 \mathrm{mmol} / \mathrm{mol})$ and $\geq 7 \%$ (53 $\mathrm{mmol} / \mathrm{mol})[5]$.
Statistical analysis Baseline characteristics were stratified by physical activity and weighted to be representative of the US population. Differences between physical activity groups were assessed using independent Student's $t$ tests and $\chi^{2}$ tests. HR and 95\% CI for all-cause and CVD mortality risk stratified by physical activity group and $\mathrm{HbA}_{1 \mathrm{c}}$ level were estimated by Cox proportional hazard regressions. Inactive individuals with $\mathrm{HbA}_{1 \mathrm{c}}<5.7 \%$ (39 $\mathrm{mmol} / \mathrm{mol}$ ) were the referent. Models were adjusted for age, sex, ethnicity, income, smoking status, fatty diet, alcohol intake, history of CVD, hypertension, total cholesterol, HDL cholesterol, BMI and insulin resistance (HOMA). Analyses were performed using SAS version 9.2 (SAS Institute, Inc., Cary, NC, USA).

\section{Results}

Baseline characteristics stratified by physical activity group are presented in Table 1. The majority of the population was categorised as active, with only $20 \%$ reporting no leisure-time physical activity. Active individuals had a lower BMI, lower total cholesterol, were more insulinsensitive and were less likely to have CVD, type 2 diabetes, or hypertension or be on medications for these conditions $(p<0.05)$.

During follow-up of $13.4 \pm 3.9$ years, there were 2,463 deaths, with 1,113 due to cardiovascular causes. Physical activity was associated with a decreased risk of all-cause (HR 0.74 [95\% CI 0.67, 0.81]) and CVD (HR 0.71 [95\% CI $0.62,0.82]$ ) mortality. In comparison with individuals with normal glycaemic control, higher levels of $\mathrm{HbA}_{1 \mathrm{c}}$ were associated with increased mortality risk. $\mathrm{HbA}_{1 \mathrm{c}} \geq 7 \%$ $(53 \mathrm{mmol} / \mathrm{mol})$ was associated with the highest risk for allcause (HR 1.54 [95\% CI 1.30, 1.82]) and CVD (HR 1.93 [95\% CI $1.52,2.45])$ mortality.

Figure 1 presents the combined effects of physical activity and $\mathrm{HbA}_{1 \mathrm{c}}$ level on all-cause and CVD mortality risk. Across all levels of $\mathrm{HbA}_{1 \mathrm{c}}$, active individuals had a lower risk of mortality compared with inactive individuals with the same $\mathrm{HbA}_{1 \mathrm{c}}$ level. In addition, individuals with $\mathrm{HbA}_{1 \mathrm{c}} \geq 7 \%(53 \mathrm{mmol} / \mathrm{mol})$ were not at an increased risk for all-cause mortality if they were physically active. For CVD mortality, active individuals with $\mathrm{HbA}_{1 \mathrm{c}} \geq 7 \%$ (53 mmol $/ \mathrm{mol}$ ) were still at an increased risk compared with inactive euglycaemic individuals; however, their risk for CVD death was substantially lower than their inactive counterparts with the same level of dysglycaemia (HR 1.38 [95\% CI 1.03, 1.84] vs HR 1.98 [95\% CI 1.34, 2.92]).

When physical activity was divided by intensity (electronic supplementary material [ESM] Table 1), light and moderate/vigorous activity was still protective against all- 
Table 1 Baseline characteristics according to physical activity level

\begin{tabular}{|c|c|c|}
\hline \multirow[t]{2}{*}{ Characteristic } & \multicolumn{2}{|c|}{ Physical activity level } \\
\hline & $\begin{array}{l}\text { Inactive } \\
(n=2,107)\end{array}$ & $\begin{array}{l}\text { Active } \\
(n=8,245)\end{array}$ \\
\hline Age (years) & $50.0 \pm 0.1$ & $44.4 \pm 0.5^{*}$ \\
\hline Men $(\%)$ & 34.0 & $51.2 *$ \\
\hline \multicolumn{3}{|l|}{$\mathrm{HbA}_{1 \mathrm{c}}(\%)$} \\
\hline$<5.7$ & $5.1 \pm 0.1$ & $5.0 \pm 0.1 *$ \\
\hline $5.7-6.4$ & $5.9 \pm 0.1$ & $5.9 \pm 0.1$ \\
\hline $6.5-6.9$ & $6.6 \pm 0.1$ & $6.7 \pm 0.1^{*}$ \\
\hline$\geq 7.0$ & $8.8 \pm 0.1$ & $9.0 \pm 0.2$ \\
\hline \multicolumn{3}{|l|}{$\mathrm{HbA}_{1 \mathrm{c}}(\mathrm{mmol} / \mathrm{mol})$} \\
\hline$<39$ & $32.6 \pm 0.2$ & $31.8 \pm 0.2 *$ \\
\hline $39-46$ & $41.3 \pm 0.1$ & $41.1 \pm 0.1$ \\
\hline $48-52$ & $48.9 \pm 0.2$ & $49.6 \pm 0.2 *$ \\
\hline$\geq 53$ & $72.4 \pm 1.6$ & $74.7 \pm 1.8$ \\
\hline Total cholesterol (mmol/l) & $5.4 \pm 0.1$ & $5.3 \pm 0.1^{*}$ \\
\hline HDL-cholesterol (mmol/l) & $1.3 \pm 0.1$ & $1.3 \pm 0.1$ \\
\hline HOMA & $3.1 \pm 0.1$ & $2.5 \pm 0.1^{*}$ \\
\hline BMI $\left(\mathrm{kg} / \mathrm{m}^{2}\right)$ & $27.7 \pm 0.4$ & $26.6 \pm 0.1^{*}$ \\
\hline Hypertension (\%) & 39.4 & $27.3 *$ \\
\hline Pre-existing type 2 diabetes ( $\%)$ & 7.4 & $3.8^{*}$ \\
\hline Pre-existing CVD (\%) & 10.5 & $4.7^{*}$ \\
\hline Current medication use $^{\mathrm{a}}(\%)$ & 14.3 & $9.5^{*}$ \\
\hline
\end{tabular}

Data are means \pm SE

${ }^{\text {a }}$ Current medication use $=$ medication use for type 2 diabetes, CVD, dyslipidaemia or hypertension

*Significantly different from inactive $(p<0.05)$

cause and CVD mortality for any category of $\mathrm{HbA}_{1 \mathrm{c}}$, although no consistent pattern between exercise intensity and mortality risk was observed.

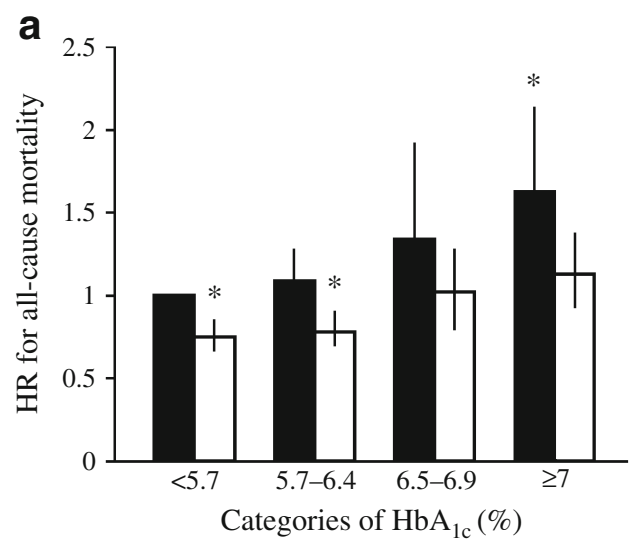

Fig. 1 The joint association of physical activity and $\mathrm{HbA}_{1 \mathrm{c}}$ level with all-cause (a) and CVD (b) mortality risk. Black bars indicate inactive; white bars indicate active. Models adjusted for age, sex, income, ethnicity, smoking status, alcohol intake, dietary fat intake, history of CVD, hypertension, total cholesterol, HDL-cholesterol, BMI and insulin resistance (HOMA). *Significantly different from referent

\section{Conclusions}

This analysis demonstrates that physical activity is associated with both lower all-cause and CVD mortality risk across the range of glycaemic control. Our findings suggest that engaging in physical activity and achieving normal levels of glycaemic control may both be important factors for the prevention of early mortality. If optimal glycaemic control is not achieved, regular exercise may still have a protective effect on overall mortality, independent of traditional metabolic risk factors. Alternatively, if physical activity is not achieved, then good glycaemic control may be critical in lowering morality risk.

A physically active lifestyle is associated with decreased risk of mortality in individuals with normoglycaemia as well as those with impaired glucose tolerance or type 2 diabetes $[6,7]$. Given that increased $\mathrm{HbA}_{1 \mathrm{c}}$ levels are associated with an increased risk of cardiovascular events and mortality in individuals with [8] and without [2] type 2 diabetes, it is important to examine whether active individuals have reductions in mortality risk, even if $\mathrm{HbA}_{1 \mathrm{c}}$ levels remain elevated. The current findings extend previous research and demonstrate that for all levels of $\mathrm{HbA}_{1 \mathrm{c}}$, active individuals may receive substantial mortality risk reductions compared with inactive individuals with the same $\mathrm{HbA}_{1 \mathrm{c}}$ level. Interestingly, this protective effect of physical activity was observed after adjustment for traditional risk factors such as insulin resistance, BMI and the lipid profile. Our results also highlight that physical activity is associated with a reduction in mortality risk above and beyond normalisation of glycaemic control. The physiological mechanisms responsible for the activity-induced reduction in mortality risk remains unclear. However, animal models suggest that aerobic exercise can induce adaptations

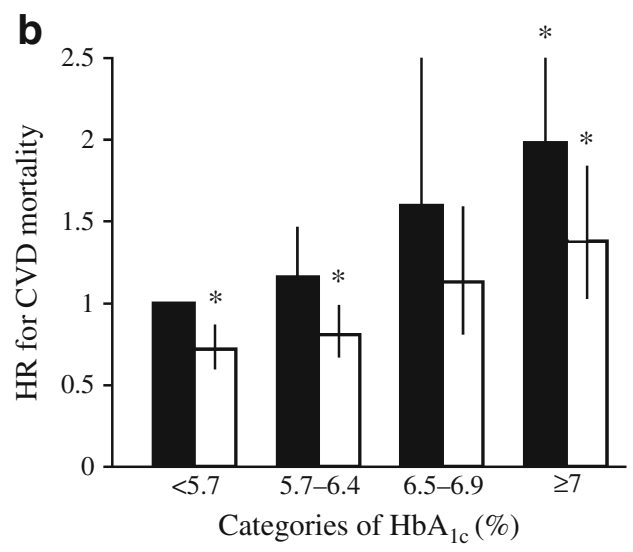

category: $\mathrm{HbA}_{1 \mathrm{c}}<5.7 \%$ inactive $(p<0.05)$. All-cause mortality: activity $\times \mathrm{HbA}_{1 \mathrm{c}}: p>0.05$, activity main effect: $p<0.05$; $\mathrm{HbA}_{1 \mathrm{c}}$ main effect: $p<0.05$. CVD mortality: activity $\times \mathrm{HbA}_{1 \mathrm{c}}: p>0.05$, activity main effect: $p<0.05$; $\mathrm{HbA}_{1 \mathrm{c}}$ main effect: $p<0.05$. To convert values for $\mathrm{HbA}_{1 \mathrm{c}}$ in $\%$ to $\mathrm{mmol} / \mathrm{mol}$, subtract 2.15 and multiply by 10.929 
that restore mitochondrial alterations associated with ageing and metabolic diseases [9]. In addition, physical activity can increase skeletal muscle mass, insulin sensitivity, and cardiac and endothelial function; all factors that may increase longevity [10]. Conversely, it is plausible that the lack of metabolic diseases allowed the individuals to be physically active thus avoiding early mortality events.

In the clinical setting, there is a large emphasis for all patients with diabetes mellitus to achieve a $\mathrm{HbA}_{1 \mathrm{c}}<7 \%$ (53 $\mathrm{mmol} / \mathrm{mol}$ ) as a way to minimise microvascular and macrovascular complications and prevent early death [5]. Our findings suggest that individuals with good glycaemic control are protected compared with those with poor glycaemic control, whether they are active or not. Active individuals with $\mathrm{HbA}_{1 \mathrm{c}} \geq 7 \%(53 \mathrm{mmol} / \mathrm{mol})$ were not at an increased risk for all-cause mortality and their risk for death was comparable with inactive individuals with normal glycaemic control. This suggests that engaging in an active lifestyle may provide similar protection against all-cause mortality as achieving glycaemic targets. Although active individuals with $\mathrm{HbA}_{1 \mathrm{c}} \geq 7 \%(53 \mathrm{mmol} / \mathrm{mol})$ still had significantly increased risk for CVD mortality compared with euglycaemic individuals, their risk was substantially lower than their inactive counterparts with the same level of dysglycaemia. It is unclear what intensity of physical activity is required to obtain these benefits, but we provide preliminary evidence that even light physical activity may be sufficient for individuals with $\mathrm{HbA}_{1 \mathrm{c}} \geq 7 \%$. These results highlight the clinical importance of prescribing physical activity for not only improving glycaemic control, but perhaps for also preventing early death.

Limitations of the current analysis warrant mention. Physical activity levels were based on self-reported questionnaire data, which did not include non-leisure-time activity (i.e. labour, transportation) or the duration of time spent in each activity, hence participants' physical activity levels are subject to misclassification. Thus, we were unable to determine the optimal volume and intensity of physical activity necessary to lower mortality risk in this population. Also, given the cross-sectional design of the study, causality cannot be inferred. Nevertheless, a long length of follow-up with a large number of deaths and the use of a large sample representative of the general US population are significant strengths to the study.

In conclusion, this study demonstrates that physical activity is associated with lower all-cause and CVD mortality risk in individuals across all levels of $\mathrm{HbA}_{1 \mathrm{c}}$, independent of traditional metabolic risk factors. Therefore, physical activity should be strongly recommended for the prevention of early death in all individuals, especially those with poor glycaemic control.

Acknowledgements The Centers for Disease Control and Prevention (CDC) funded and conducted the NHANES III study with mortality follow-up and made the data publicly available. The CDC had no role in this study. The authors had full access to all of the data in the study and take responsibility for the integrity of the data analysis. The authors acknowledge the following granting agencies for their support: The Canadian Diabetes Association (M.C. Riddell), and Networks of Centres of Excellence of Canada and Mitacs-Accelerate (J.L. Kuk).

Contribution statement All of the authors listed helped with the conception/design of the study as well as the analysis and/or interpretation of these data. All authors assisted in either drafting the article and/or revising it critically. All authors approved the final version of the manuscript for publication.

Duality of interest The authors declare that there is no duality of interest associated with this manuscript.

\section{References}

1. Gerstein HC (2009) Dysglycemia and cardiovascular risk in the general population. Circulation 119:773-775

2. Selvin E, Steffes MW, Zhu H et al (2010) Glycated hemoglobin, diabetes, and cardiovascular risk in nondiabetic adults. $\mathrm{N}$ Engl $\mathrm{J}$ Med 362:800-811

3. Colberg SR, Sigal RJ, Fernhall B et al (2010) Exercise and type 2 diabetes: the American College of Sports Medicine and the American Diabetes Association: joint position statement. Diabetes Care 33:e147-e167

4. Matthews DR, Hosker JP, Rudenski AS, Naylor BA, Treacher DF, Turner RC (1985) Homeostasis model assessment: insulin resistance and beta-cell function from fasting plasma glucose and insulin concentrations in man. Diabetologia 28:412-419

5. American Diabetes Association (2011) Standards of medical care in diabetes-2011. Diabetes Care 34(Suppl 1):S11-S61

6. Hu G, Jousilahti P, Barengo NC, Qiao Q, Lakka TA, Tuomilehto J (2005) Physical activity, cardiovascular risk factors, and mortality among Finnish adults with diabetes. Diabetes Care 28:799-805

7. Batty GD, Shipley MJ, Marmot M, Smith GD (2002) Physical activity and cause-specific mortality in men with type 2 diabetes/ impaired glucose tolerance: evidence from the Whitehall study. Diabet Med 19:580-588

8. Eeg-Olofsson K, Cederholm J, Nilsson PM et al (2010) New aspects of $\mathrm{HbA}_{1 \mathrm{c}}$ as a risk factor for cardiovascular diseases in type 2 diabetes: an observational study from the Swedish National Diabetes Register (NDR). J Intern Med 268:471-482

9. Safdar A, Bourgeois JM, Ogborn DI et al (2011) Endurance exercise rescues progeroid aging and induces systemic mitochondrial rejuvenation in mtDNA mutator mice. Proc Natl Acad Sci U S A 108:4135-4140

10. Warburton DE, Nicol CW, Bredin SS (2006) Health benefits of physical activity: the evidence. CMAJ 174:801-809 